\title{
Fatores estressantes para familiares de pacientes criticamente enfermos de uma unidade de terapia intensiva
}

Stressful factors for families of critically ill patients of the intensive care unit

Jaquilene Barreto da Costa', Claudia Regina Felicetti', Claudia Rejane L. de Macedo Costa', Dalas Cristina Miglioranza', Erica Fernanda Osaku', Gelena Lucinéia G. da Silva Versa', Joanito Solstoski', Péricles Almeida D. Duarte', Silvana Triló Duarte', Suely Mariko Ogasawara', Sheila Taba'

\section{RESUMO}

Objetivo: Identificar os principais estressores ambientais, conforme a percepção de familiares de pacientes internados em uma UTI-G de adultos de um hospital público universitário. Método: Estudo transversal descritivo com familiares de pacientes gravemente enfermos internados na UTI de um hospital escola. Para a coleta de dados, utilizou-se uma escala contendo 25 itens relacionados a eventos da referida UTI. Para análise dos resultados, as respostas foram classificadas como estressores ambientais, referentes à equipe, ao paciente e à visita. Resultados: Participaram 53 familiares, 67,9\% mulheres, com média de idade de 39,7 anos. Os fatores relacionados ao paciente foram mais estressantes do que aqueles referentes à equipe e ao ambiente $(p<0,005)$. Os eventos mais estressantes para os familiares estiveram relacionados à insegurança e ao medo quanto ao estado clínico do paciente, dentre esses, o motivo e o tempo de internação e ver o paciente em coma. Conclusão: $A$ internação de um parente próximo na UTI foi considerada pelos familiares que efetivamente participaram desse processo um evento estressante. Todos os participantes indicaram pelo menos um evento causador de estresse capaz de provocar diferentes reações emocionais. Conhecer tais eventos pode facilitar as estratégias de humanização hospitalar, propiciando alternativas para reduzir os níveis de estresse e alterações psiquiátricas subsequentes.

\begin{abstract}
Objective: To identify main environment stressors according to the perception of relatives of patients hospitalized in an adult ICU in a university public hospital. Method: It is a transversal descriptive study involving family members of critically ill patients hospitalized in an ICU of a school hospital. To collect data we used a 25-item scale related to the referred ICU. For result analysis, answers were classified as environmental stressors concerning the team, patients, and visits. Results: There were 53 family-member participants where $67.9 \%$ were women averaging 39.7 years of age. Factors related to patients were more stressing than those concerning the hospital team and the environ-
\end{abstract}

1 Universidade Estadual do Oeste do Paraná (Unioeste), Hospital Universitário do Oeste do Paraná, Setor de Psicologia. 


\section{Keywords}

Intensive care units, psychological stress, family. ment ( $p<0.005)$. The most stressed events for the family members were related to uncertainty and fear of the clinical state of the patient, among them reason and length of hospital stay, and witness the patients in a coma. Conclusion: Hospital ICU stay of a relative was considered by the close to kin, who actually participated in the process, a stressful event. All participants indicated at least one event that causes stress as capable of provoking diverse emotional reactions. Knowing such events can favor hospital humanization strategies thus providing alternatives to reduce stress levels and subsequent psychiatrical disorder.

\section{INTRODUÇÃO}

A admissão em uma unidade de terapia intensiva geral (UTI-G) é um evento estressante tanto para o paciente quanto para os familiares, sendo este caracterizado como uma situação tensa, fisiológica e/ou psicológica, podendo afetar as pessoas em todas as suas dimensões.

Muitos estudos têm pesquisado o estresse do paciente relacionado ao ambiente da UTT1 ${ }^{-4} \mathrm{e}$, em sua grande maioria, o enfoque recai sobre a avaliação dos eventos estressores na perspectiva da família e da equipe ${ }^{5,6}$. No entanto, no caso de pacientes gravemente enfermos, frequentemente sedados e imobilizados, o familiar, como também a própria equipe, pode apenas supor o que causa dor ou desconforto ao paciente, angústia e sofrimento psíquico 7 .

O estresse tem sido relacionado a sensações de tensão, ansiedade, medo e desconforto caracterizado por alterações psicofisiológicas que ocorrem quando o indivíduo é forçado a enfrentar situações que estão além de suas habilidades de enfrentamento ${ }^{8}$. Situações específicas como a presença de tubos na boca e/ou nariz, dor, comprometimento do sono, não ter controle de si mesmo, limitação de movimentos e não ter explicação sobre o seu tratamento são descritas na literatura como os itens mais associados ao desenvolvimento de estresse pelos pacientes ${ }^{4,5}$.

A internação em UTI gera também um alto grau de estresse e ansiedade na família9. A gravidade clínica do paciente e a impossibilidade em se comunicar têm sido descritas como fatores de impacto no grau de estresse da família. Dessa forma, a avaliação de eventos causadores de estresse e sofrimento psíquico deve assumir um enfoque diferente, sendo necessária uma atenção direcionada aos familiares.

A gravidade do quadro clínico, a alteração do nível de consciência e a ausência de comunicação implicam a impossibilidade de tomada de decisões pelo paciente ${ }^{10}$, transferindo para os familiares um papel central durante o tratamento, reabilitação e cuidados empregados após a alta. É necessário, portanto, detectar e minimizar o impacto de eventos estressores na saúde mental dos familiares.

A literatura tem apontado altos níveis de ansiedade e sintomas depressivos entre membros de familiares de pacientes internados em UTI11-13, já que a internação nessa unidade envolve uma ameaça iminente à integridade física do paciente, podendo se configurar como um potencial evento traumático causador de estresse emocional. Nessa linha, outros estudos ${ }^{14-16}$ têm descrito a presença de reações de estresse pós-traumático meses após o internamento na UTI, e pelos resultados encontrados sugere-se a necessidade de desenvolver estratégias preventivas para minimizar o desencadeamento de alterações emocionais em familiares a curto e longo prazo.

Diante disso, ao longo dos últimos anos os cuidados têm sido estendidos aos familiares, proporcionando atenção especial às suas necessidades emocionais, reduzindo os riscos de transtornos psíquicos subsequentes ${ }^{7-17}$. Conhecer os fatores associados ao desencadeamento de estresse dentro do ambiente de terapia intensiva pode ser útil para ampliar as estratégias de atenção aos familiares com vistas à prevenção de possíveis alterações emocionais.

Este estudo teve como objetivo avaliar os principais estressores ambientais, conforme a percepção de familiares de pacientes internados em uma UTI-G de adultos de um hospital público universitário.

\section{MÉTODO}

Estudo transversal descritivo com familiares de pacientes internados em uma UTI-G de um hospital universitário público. A UTI em estudo é referência de alta complexidade para 26 municípios da região, conta com nove leitos destinados a pacientes adultos acometidos de patologias clínicas diversas (clínico-cirúrgicas, trauma e neurológicos) e atende em média 350 pacientes ao ano.

As visitas de familiares nesta UTI são realizadas duas vezes ao dia, em períodos de 30 minutos, seguidas de entrevista com o médico plantonista. É permitida a entrada de duas pessoas durante cada período de visita, sendo estimulada a presença de familiares como acompanhantes dentro da UTI, exclusivamente no caso de pacientes em processo de desmame ou já fora da ventilação mecânica. No caso de pacientes sedados e gravemente enfermos, não há acompanhantes continuamente e a interface com a equipe se dá durante ou após a visita, na entrevista com o médico plantonista.

Participaram deste estudo familiares de pacientes internados no período de junho a dezembro de 2008 e que 
preencheram os seguintes critérios de inclusão: parentes de primeiro grau (pais, irmãos, cônjuges, filhos), com familiar internado na UTI por mais de 36 horas e em ventilação mecânica no dia da visita, que tivesse realizado pelo menos duas visitas até o momento da entrevista, maiores de 18 anos, e que consentiram sua participação no estudo. Foram excluídos familiares de pacientes com diagnóstico de morte cerebral e doença terminal.

Para a coleta de dados, foi utilizado um formulário contendo dados sociodemográficos e clínicos do paciente, ficha de identificação do entrevistado e uma escala contendo 25 itens referentes a eventos relacionados à UTI considerados como estressantes.

Atualmente, não há instrumentos nacionais padronizados para avaliar os eventos estressores relacionados ao contexto de tratamento intensivo, causadores de estresse na família. Diante disso, os pesquisadores desenvolveram uma escala baseada no conteúdo de alguns itens da Escala de Estressores em Unidade de Terapia Intensiva (Intensive Care Unit Environmental Stressor Scale - ICUESS), validada por Ballard ${ }^{18}$ e por Nastasy ${ }^{19}$, traduzida e adaptada culturalmente por Novaes et al. ${ }^{4}$, composta por 40 itens para identificar os principais eventos percebidos como estressantes para o paciente. Para a construção do instrumento, realizou-se uma adaptação da referida escala, considerando as especificidades do tratamento intensivo e os eventos estressores que poderiam ser aplicados na avaliação dos familiares. Para a elaboração do instrumento, foram percorridas duas etapas: análise do instrumento por um grupo de profissionais com experiência na área de terapia intensiva (3 médicos, 2 psicólogos, 1 assistente social, 2 enfermeiros, 3 fisioterapeutas e 1 fonoaudióloga), com vistas a sugerir e verificar a forma e apresentação dos itens quanto à clareza, facilidade de leitura, abrangência do conteúdo e relevância. Em seguida, realizouse um estudo piloto para verificar a aplicabilidade na população-alvo. No estudo piloto, foram convidados, aleatoriamente durante o horário de visita da UTI estudada, 13 sujeitos para responder à escala, indicando os eventos causadores de estresse de acordo com a pontuação estabelecida. Desses, seis eram homens e sete, mulheres, com idade variando entre 20 e 65 anos, sendo informados do objetivo de sua participação. Após realização do estudo piloto, verificou-se que os itens eram compreensíveis e que o instrumento possibilitou a identificação de eventos relacionados à UTI indicados pelos familiares como causadores de estresse. A versão final do instrumento ficou constituída por 25 itens, agrupados em quatro domínios: os eventos estressores relacionados ao ambiente, relativos ao paciente, a interação equipe/família e referentes à visita.

Após a avaliação do instrumento pelo grupo de profissionais, ajustamento de alguns itens e realização do estudo piloto, teve início a pesquisa com os familiares de pacientes internados durante o período do estudo.
A aplicação da escala foi realizada individualmente na sala de espera, logo após o horário de visita. Os participantes determinaram o grau de estresse de cada item por meio de uma escala de valores pontuando de 0 a 3 , sendo o valor zero (0) considerado como não estressante, um (1), pouco estressante, dois (2), estressante e três (3), muito estressante. Foi entrevistado apenas um familiar por paciente e, em nenhum dos casos, os familiares estavam na rotina de permanência, como acompanhantes.

Para a análise dos dados, os fatores estressores foram classificados de acordo com as quatro categorias: estressores ambientais ( 8 itens), relacionados ao paciente (9 itens), relativos à interação entre equipe/família (4 itens) e referentes à visita ou rotinas administrativas (4 itens). O escore de todos os itens foi baseado nas respostas dadas, variando entre não estressante (1 ponto), pouco estressante (2 pontos), estressante (3 pontos) e muito estressante (4 pontos). A análise dos estressores foi feita a partir do ranking estabelecido pelo escore de todos os itens.

Os dados foram armazenados no programa Microsoft ${ }^{\oplus}$ Excel $^{\oplus}$ e, para análise e interpretação dos resultados, foi utilizado o programa Statistica 8.0. Foi realizada análise de porcentagem simples para caracterizar a amostra de acordo com as variáveis clínicas e sociodemográficas. Realizaram-se estatística descritiva e análise de variância e teste-T, aceitando-se um $p<0,05$. Os resultados são mostrados em forma de tabelas e apresentados em frequência absoluta e relativa. Dados contínuos são apresentados como média e desviopadrão, quando indicados, e dados categóricos em número absoluto e porcentagem.

O estudo foi desenvolvido em conformidade com o preconizado pela Resolução no 196/96, do Conselho Nacional de Saúde, e o projeto foi apreciado pelo Comitê Permanente de Ética em Pesquisas envolvendo Seres Humanos da Universidade Estadual do Oeste do Paraná. A avaliação de todos os pacientes incluídos no estudo ocorreu após a assinatura do termo de consentimento livre e esclarecido.

\section{RESULTADOS}

Durante o período de estudo, 187 pacientes foram admitidos na UTI de adultos, sendo 172 por período maior do que 36 horas. Desses, 119 foram excluídos, seguindo critérios previamente determinados.

Dos 53 familiares participantes, $67,9 \%$ eram do sexo feminino e a idade deles variou entre 18 e 69 anos, com média de $39,7 \pm(12,7)$ anos. A maioria dos entrevistados $(66,1 \%)$ tinha completado o ensino fundamental e 56,6\% desenvolviam atividade laboral remunerada. Quanto ao grau de parentesco, 33,9\% dos entrevistados eram cônjuges, e uma grande proporção (62,3\%) afirmou que, após a internação, seria o cuidador do paciente. A maioria dos entrevistados (62,3\%) 
residia na mesma cidade onde estava internado o paciente e, no momento da entrevista, 58,5\% dos familiares tinham realizado em média cinco visitas.

Quanto aos pacientes, 67,3 eram do sexo masculino e, do total, 51\% foram admitidos por causa de condições clínicas diversas, especialmente a sepse (38,8\%), seguidas daqueles com trauma $(34,7 \%)$ e por condições neurológicas (14,3\%). O tempo médio de permanência na UTI foi de 10,0 dias $( \pm 8,5)$, variando entre 2 e 37 dias; $47,8 \%$ dos pacientes ficaram internados na UTI por mais de sete dias e mais da metade $(55,8 \%)$ permaneceu em ventilação mecânica por mais de dois dias, com um tempo médio de 7,2. O escore médio do índice de APACHE II foi 20,2 (mortalidade esperada de cerca de $36 \%$ ). Não houve diferenças significantes entre os pacientes incluídos no estudo e os não incluídos em relação a idade, sexo, gravidade (mensurado pelo escore APACHE II) e causas de admissão. Dados epidemiológicos e sociais dos pacientes e dos familiares envolvidos no estudo são mostrados na tabela 1.

Tabela 1. Características sociodemográficas dos pacientes e membros das famílias

\begin{tabular}{lll}
\hline & Pacientes & Familiares \\
\hline Sexo masc. (\%) & $67,3 \%$ & $32,1 \%$ \\
Idade (média + DP) & $42,08+17,28$ & $39,7+12,7$ \\
Idade média (mín.-máx.) & $42,08(17-87)$ & $39,7(18-69)$ \\
Escolaridade & & \\
Analfabeto & $11,3 \%$ & $01,9 \%$ \\
$1^{\circ}$ grau & $64,2 \%$ & $66,1 \%$ \\
$2^{\circ}$ grau & $11,3 \%$ & $22,6 \%$ \\
Superior & $03,8 \%$ & $09,4 \%$ \\
Não respondeu & $09,4 \%$ & 0 \\
Ocupação & & \\
Trabalha & $56,6 \%$ & $56,6 \%$ \\
Não trabalha & $28,3 \%$ & $35,9 \%$ \\
Aposentado & $15,1 \%$ & $07,5 \%$ \\
Grau de parentesco & & \\
Cônjuge & - & $33,9 \%$ \\
Filho(a) & - & $22,7 \%$ \\
Pai/mãe & - & $18,9 \%$ \\
Irmã(o) & - & $24,5 \%$ \\
\hline
\end{tabular}

A possibilidade de pontuação da escala variava entre 25 e 100 pontos, e quanto maior o valor do somatório dos itens, mais estressantes eram os eventos analisados pela escala. $\mathrm{Na}$ amostra estudada, foram obtidos valores entre 56 e 150 pontos referentes às quatro notas da escala $(0,1,2,3)$, tendo os itens relacionados ao paciente alcançado a maior média $(127,1$ pontos). Na sequência ficou o agrupamento dos itens referentes à visita (103 pontos), seguidos da categoria ambiente ( 90,4 pontos) e equipe ( 74,2 pontos). Pelas medidas descritivas referentes aos quatro domínios da escala, constatou-se que as médias obtidas no agrupamento dos itens de cada categoria foram relativamente próximas $(0,16 ; 0,36 ; 0,2$ e 0,28). A tabela 2 mostra o ranking dos fatores estressores referidos pelos familiares.

No geral, os familiares indicaram pelo menos um evento percebido como estressante referente à internação de um familiar na UTI. Os eventos percebidos como muito estressantes, pontuados como nível três, foram: ver o paciente em coma/sedado $(66,1 \%)$, entubado $(58,5 \%)$, seguidos do motivo de internação $(56,6 \%)$ e o paciente não conseguir falar (51\%).

Os itens relacionados ao contato com a equipe da UTI foram os que receberam os mais baixos escores de estresse. Apenas o item "Não conhecer os membros da equipe" foi percebido como sendo um fator estressante. Os fatores ambientais que também tiveram baixa pontuação, entre os mais citados, foram: "O ambiente da UTI" e "Ver os outros pacientes". Por outro lado, "As luzes da UTI" e "Os barulhos da UTI" foram raramente mencionados. O gráfico 1 mostra os cinco primeiros fatores estressantes com maiores e menores escores.

Os fatores relacionados à visita tiveram uma pontuação intermediária, atrás apenas dos relacionados ao paciente. A exceção foi o item "Não poder permanecer como acompanhante", que foi considerado pelos familiares como um evento muito estressante. Já os eventos considerados como muito estressante foram os itens relacionados ao paciente, entre eles os mais pontuados foram: "A causa e tempo de internação", "O paciente em coma" e "Ver o paciente entubado". Nenhum item referente à categoria ambiente e equipe esteve entre os mais citados, por outro lado, quatro dos cinco itens mais citados eram do grupo "Paciente".

Pelos resultados da análise realizada, foram observados níveis de significância estatística em relação às categorias paciente e equipe $(p<0,001)$; paciente e ambiente $(p=0,004)$. Houve uma clara diferença entre os grupos "Paciente"/"Visita" versus "Ambiente"/"Equipe", com os primeiros causando muito mais estresse aos membros das famílias do que os últimos. Os dados referentes aos grupos são mostrados no gráfico 2 .

\section{DISCUSSÃO}

A evolução da tecnologia, habilidade e experiência no manejo de pacientes criticamente enfermos, bem como a disseminação do conhecimento pela população leiga, têm levado a mudanças na abordagem por parte dos profissionais de terapia intensiva. O enfoque exclusivamente tecnicista tem sido colocado em dúvida quanto a aspectos éticos e humanísticos, e uma abordagem voltada aos interesses do paciente tem sido demonstrada ser factível e eficaz e, recentemente, tem-se dado maior atenção à assistência aos familiares ${ }^{15-17,20}$. 
Tabela 2. Ranking dos fatores estressantes de acordo com a classificação das notas de cada item das categorias: visita, paciente, equipe e ambiente

\begin{tabular}{|c|c|c|c|c|c|c|c|c|c|}
\hline \multirow[t]{2}{*}{ Categorias } & \multirow[t]{2}{*}{ Itens } & \multicolumn{4}{|c|}{$\%$ dos escores } & \multirow[b]{2}{*}{ N/A } & \multirow[b]{2}{*}{ Notas } & \multirow[b]{2}{*}{ Ranking } & \multirow[b]{2}{*}{ Média categ. } \\
\hline & & 0 & 1 & 2 & 3 & & & & \\
\hline \multirow[t]{4}{*}{ Visita } & Horário de visitas & 69,9 & 22,7 & 3,7 & 3,7 & & 75 & 21 & \\
\hline & Tempo para visita & 62,3 & 18,9 & 6,8 & 12,0 & & 90 & 15 & \\
\hline & Não ficar como acompanhante & 28,3 & 20,7 & 17,0 & 34,0 & & 136 & 05 & \\
\hline & Atraso no horário de visita & 45,3 & 18,9 & 17,0 & 18,9 & & 111 & 11 & 0,16 \\
\hline \multirow[t]{9}{*}{ Paciente } & Tempo de internação & 22,7 & 15,1 & 24,5 & 37,7 & & 147 & 02 & \\
\hline & Ver o paciente entubado & 5,6 & 15,1 & 20,8 & 58,5 & & 133 & 07 & \\
\hline & Motivo da internação & 1,9 & 13,2 & 28,3 & 56,6 & & 150 & 01 & \\
\hline & Paciente em coma & 1,9 & 9,5 & 22,6 & 66,1 & & 143 & 03 & \\
\hline & Paciente sem conseguir falar & 3,7 & 18,9 & 22,7 & 51,0 & 3,7 & 139 & 04 & \\
\hline & Paciente amarrado/contido & 11,3 & 15,1 & 9,5 & 28,3 & 35,9 & 97 & 13 & \\
\hline & Paciente amarrado a tubo & 13,2 & 15,1 & 41,5 & 30,2 & & 134 & 06 & \\
\hline & Ver o paciente sem roupa & 62,3 & 18,9 & 5,6 & 13,2 & & 90 & 14 & \\
\hline & Ver o paciente descoberto & 37,7 & 22,7 & 17,0 & 18,9 & 3,7 & 111 & 10 & 0,36 \\
\hline \multirow[t]{4}{*}{ Equipe } & Contato com a equipe & 88,7 & 11,3 & 0 & 0 & & 59 & 24 & \\
\hline & Contato com o médico & 73,6 & 15,1 & 11,3 & 0 & & 73 & 22 & \\
\hline & Informações dadas & 71,3 & 9,5 & 13,2 & 5,6 & & 81 & 20 & \\
\hline & Não conhecer a equipe & 58,5 & 0 & 13,2 & 15,1 & 13,2 & 84 & 18 & 0,2 \\
\hline \multirow[t]{8}{*}{ Ambiente } & Barulho dos aparelhos & 66,1 & 15,1 & 7,5 & 11,3 & & 87 & 16 & \\
\hline & Iluminação & 94,4 & 5,6 & 0 & 0 & & 56 & 25 & \\
\hline & Barulho do ambiente & 69,9 & 15,1 & 7,5 & 7,5 & & 81 & 19 & \\
\hline & Aparelhos ao redor do paciente & 37,7 & 20,7 & 26,5 & 15,1 & & 116 & 09 & \\
\hline & Número de pessoas na UTI & 81,1 & 13,2 & 3,7 & 1,9 & & 67 & 23 & \\
\hline & Ver os outros pacientes & 45,3 & 18,9 & 18,9 & 17,0 & & 111 & 12 & \\
\hline & 0 ambiente da UTI & 39,6 & 18,9 & 20,7 & 20,7 & & 118 & 08 & \\
\hline & Cheiros da UTI & 66,0 & 13,2 & 11,3 & 9,5 & & 87 & 17 & 0,28 \\
\hline
\end{tabular}

(1) não estressante; (2) pouco estressante; (3) estressante (4) muito estressante.

N/A: não se aplica; média categ.: médias de cada categoria (visita, paciente, equipe e ambiente).

Obs.: Nos itens com notas semelhantes, considerou-se para posição no ranking aquele com maior porcentagem na escala de valores (0-3).

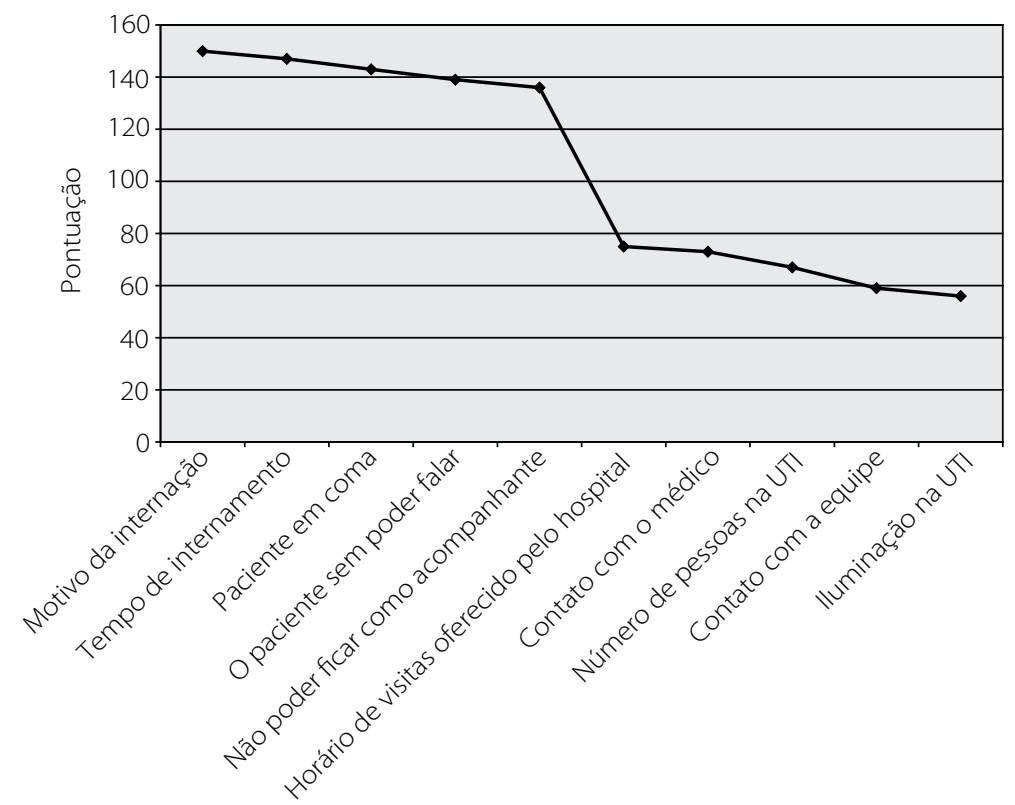

Gráfico 1. Fatores estressantes de acordo com os cinco maiores e menores escores. 


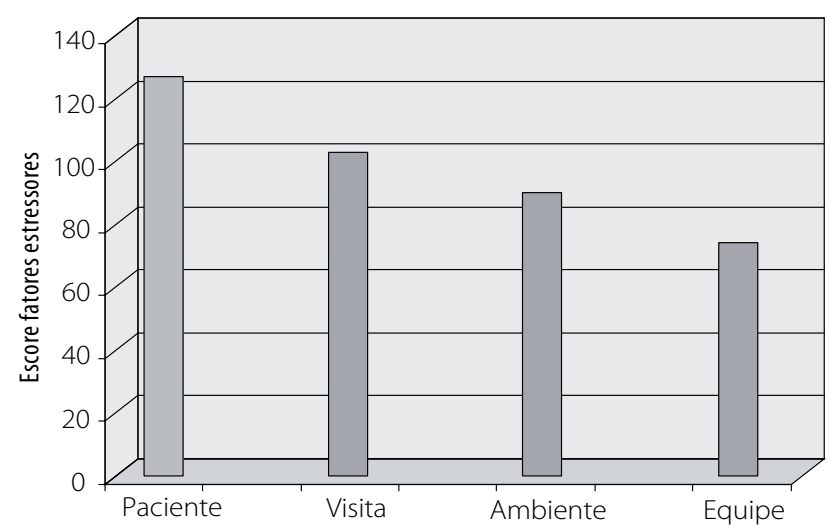

Gráfico 2. Escore médio de cada grupo de acordo com a relação entre os fatores estressantes: paciente $x$ equipe: $p<0,001$; paciente $x$ ambiente: $p=0,004$; visita $x$ equipe: $p=0,004$; visita $x$ ambiente: $p=0,093$.

Neste estudo, uma escala aplicada a familiares que efetivamente participaram do processo de visitas avaliou quais fatores são percebidos como estressantes durante a visita a um membro da família na UTI. Os resultados obtidos fornecem evidências adicionais de que a admissão de um parente próximo na UTI é considerada um evento estressante que provoca reações emocionais durante o período de internação nessa unidade. Estudos ${ }^{15,20,21}$ têm relatado que a internação em UTI, além de ser um evento estressante, gera nos familiares altos índices de sintomas de ansiedade, depressão e estresse pós-traumático, sendo essas alterações persistentes mesmo após a alta hospitalar. Os resultados do estudo de Paparrigopoulos et al. ${ }^{12}$ identificaram que $81 \%$ dos familiares avaliados apresentavam reações indicativas de estresse póstraumático seis meses após a alta hospitalar do familiar.

Neste estudo, os fatores relacionados ao ambiente, tais como barulho, luzes ou excesso de pessoas, foram relatados como eventos geradores de pouco estresse. Também, de maneira surpreendente, não foram relatados problemas em relação ao contato com a equipe ou durante a visita e entrevista com o médico, sugerindo um grau satisfatório em relação ao atendimento prestado pela equipe, principalmente nas questões relacionadas à comunicação.

Uma consideração importante em relação à baixa pontuação atribuída aos itens referentes à equipe e ao ambiente diz respeito às diferenças entre os pacientes de terapia intensiva de acordo com o tipo de UTI e/ou a gravidade dos pacientes. Os familiares e os próprios pacientes, quando em situação menos crítica (por exemplo, em unidades coronarianas ou pacientes com menor gravidade), tendem a apresentar mais queixas referentes aos fatores ambientais, tais como ruídos, iluminação, incômodo pelos aparelhos, além disso, a perda da privacidade revela-se um fator importante de estresse para essa população22,23.
Por outro lado, apesar de essa pesquisa não ter incluído dados econômicos dos familiares entrevistados, a população atendida na instituição pesquisada é constituída prioritariamente de pessoas de baixo poder aquisitivo, com baixa escolaridade. Assim, pode-se especular se as respostas poderiam ter sido influenciadas por essas características e se seriam diferentes em outras populações, com alto nível de exigência e/ou maior acesso a informações por outros meios, como a internet. Isso é sugerido pelo fato de que em nosso estudo os itens relacionados ao paciente foram percebidos como os eventos mais estressantes, o que poderia estar relacionado a uma inadequada orientação por parte da equipe, por outro lado, não houve reclamações sobre o contato com os profissionais e a entrevista médica. No entanto, apesar da garantia de sigilo pelo pesquisador, pode ter havido um receio de que uma eventual "queixa" poderia constranger ou provocar represálias.

A impossibilidade de permanência na UTI como acompanhante ao lado do paciente foi descrita como sendo um evento estressante. Em relação a esse item "Não poder ficar como acompanhante", a política da UTI é de duas visitas diárias com duração de 30 minutos e, como regra, não há permanência de familiares enquanto o paciente permanecer em condições críticas, ou seja, sedado ou instável do ponto de vista respiratório ou hemodinâmico. Em geral, a presença dos familiares é requisitada para permanecer como acompanhante quando da melhora clínica e/ou retirada da sedação.

A análise desses dados permite considerar medidas que possam reduzir o nível de estresse das famílias e assim possibilitar uma melhor compreensão acerca das rotinas da UTI. Uma explicação mais detalhada para os familiares sobre os motivos que levam a essa rotina poderia reduzir o estresse percebido por parte dos familiares pela não permanência como acompanhante durante essa fase crítica do paciente.

Ainda em relação ao item "Não poder ficar como acompanhante", que é também considerado um dos principais estressores referido pelo paciente, alguns estudos ${ }^{19,24,25}$ têm indicado como necessário um plano individualizado de visitas para minimizar a dificuldade de separação da família, com vistas à melhoria da assistência e redução da ansiedade do paciente e da família.

Neste estudo, o grande motivo de preocupação para os familiares residiu na insegurança sobre as reais condições do paciente, particularmente sobre o diagnóstico e prognóstico, bem como o nível de consciência rebaixado e a percepção de estar amarrado e/ou "preso" por meio dos vários tubos, fios e cateteres. Estudos ${ }^{5,6}$ que têm procurado avaliar o estresse dos pacientes na perspectiva dos familiares têm demonstrado que tanto os familiares quanto a equipe têm uma percepção ou previsão equivocadas em relação à intensidade e aos fatores causadores de estresse percebido pelo paciente. Esses estudos identificaram que os principais 
estressores para o paciente foram: ver a família por apenas alguns minutos, tubos no nariz e/ou boca e não ter controle de si mesmo. Já na perspectiva dos familiares, os eventos mais estressantes foram: não conseguir dormir, ter dor e estar dependente de tubos; e na visão da equipe, ter dor e o ruído dos aparelhos e do ambiente foram indicados como os fatores causadores de estresse para o paciente.

Um importante aspecto na avaliação do impacto da UTI e da doença crítica sobre os pacientes e seus familiares é a condição socioeconômica e cultural. Tais variáveis, junto com diferenças étnicas e religiosas, podem levar a dificuldade de percepção e reações diversas, ou mesmo ser causadoras de conflitos ${ }^{26,27}$. No Brasil, particularmente, as diferenças socioeconômicas são gritantes, exigindo cuidadosa interpretação de dados epidemiológicos em virtude de características peculiares de cada população estudada. Assim, dados coletados de hospitais privados, por exemplo, podem não refletir fielmente à realidade de uma população atendida em hospital público, e vice-versa.

Um estudo ${ }^{23}$ nacional que avaliou a percepção de fatores estressantes na UTI pelos familiares identificou que fatores econômicos e sociais aparentemente têm impacto significativo nas respostas. A população atendida em hospitais públicos brasileiros é em geral muito diferente daquela de hospitais privados, no que se refere ao poder aquisitivo e condições socioculturais. Assim, imagina-se que os resultados de uma pesquisa de fatores estressantes sejam diferentes entre esses dois grupos. Os resultados do presente estudo também demonstram que a pontuação atribuída aos diferentes estressores esteve relacionada com características individuais, como nível de instrução mais elevado, sexo feminino, número de visitas e cônjuge (esposa). Familiares com essas características tenderam a indicar uma pontuação maior na escala de eventos estressores, principalmente nos itens relacionados ao paciente (ver o paciente entubado, amarrado a tubos e em coma), além disso, a maioria dos familiares, independentemente de características individuais, indicou como principal estressor o motivo e o tempo de internação, provavelmente em virtude de que uma grande proporção dos pacientes admitidos foi devida a trauma, decorrente de acidentes automobilísticos e estavam em condição crítica.

Outros estudos ${ }^{28,29}$ também apontam para a influência de que algumas características dos familiares estariam relacionadas às percepções individuais de cada grupo familiar, como no estudo ${ }^{28}$ que avaliou o nível de satisfação de familiares com pacientes internados em UTI, verificando que um menor grau de satisfação esteve relacionado com o sexo, grau de instrução e número de visitas realizadas.

Um grande diferencial neste estudo é que todos os pacientes estavam, no momento da entrevista, com o familiar em situação extremamente crítica, sedados e submetidos à ventilação mecânica invasiva; isso é evidenciado pelo escore médio de APACHE, portanto retrata o perfil de pacientes in- ternados em UTIs de instituições públicas, diferenciando-se de dois outros estudos prévios ${ }^{4,5,23}$, em que o pré-requisito para participação no estudo era que o paciente estivesse consciente, lúcido e em respiração espontânea.

Além disso, a tentativa de avaliar os eventos estressores dentro do ambiente de terapia intensiva deve envolver não apenas o que a família pensa sobre o estresse do paciente, mas também entender quais eventos experimentados durante a internação de familiar na UTI poderão acarretar manifestações emocionais nos familiares ${ }^{20,21,24}$. Portanto, considerar as respostas emocionais dos familiares poderá ajudar em um melhor atendimento ${ }^{19,30}$, manter a humanização do serviç ${ }^{30}$ e reduzir as complicações psiquiátricas a curto e longo prazo $^{31}$, bem como poderá ser um poderoso instrumento em um programa de melhoria na qualidade da assistência ${ }^{31,32}$. Isso é particularmente importante em um contexto clínico, como o do atual estudo, em que o paciente encontra-se em coma ou sedado e com alto risco de morte. Muitos desses pacientes irão morrer na UTI sem que a equipe possa ter uma real avaliação sobre a adequação do tratamento, principalmente no que se refere às necessidades dos familiares, portanto a única interface de ligação pode ser a família. Essa abordagem leva a uma nova concepção de atendimento em terapia intensiva, centrado também na família ${ }^{17,33}$.

Algumas limitações devem ser ponderadas. Uma delas refere-se à ausência de itens no instrumento relacionados aos cuidados de fim de vida. Referente a isso, é de interesse que familiares de pacientes que, porventura, venham a morrer na UTI tenham demonstrado satisfação em relação à assistência prestada ao paciente e familiar. Além disso, o fato de o paciente, no momento da entrevista, permanecer internado na UTI pode ter intimidado alguns dos respondentes, os quais podem ter minimizado os fatores relacionados ao ambiente ou à interface com a equipe.

\section{CONCLUSÕES}

Neste estudo realizado em um hospital universitário com uma população de menor condição socioeconômica, os fatores estressantes para os familiares visitantes de pacientes criticamente enfermos com alto risco de morte estiveram relacionados com o estado geral do paciente e o seu sofrimento. O ambiente e as rotinas de visita foram indicados como menos geradores de estresse para essa população. Conhecer a população atendida e os fatores tidos como estressantes para os familiares pode facilitar as estratégias de humanização hospitalar, propiciando alternativas para reduzir os níveis de estresse e alterações psiquiátricas subsequentes. Estudos são necessários para avaliar a eficácia de estratégias que contribuam para a diminuição de eventos considerados como estressantes pelos familiares no contexto das unidades de cuidados intensivos. 


\section{CONFLITOS DE INTERESSE}

Os autores declaram não ter quaisquer conflitos de interesse.

\section{REFERÊNCIAS}

1. Williams CMA. The identification of family members' contribution to patients' care in the intensive care unit: a naturalistic inquiry. Nurs Crit Care. 2005;10(1):6-14.

2. Lee LYK, Lau YL. Immediate needs of adult family members of adult intensive care patients in Hong Kong. J Clin Nurs. 2003;12:490-500.

3. Holden J, Harrison L, Johnson M. Families, nurses and intensive care patients: a review of the literature. J Clin Nurs. 2002;11:140-8.

4. Novaes MA, Aronovich A, Ferraz MB, Knobel E. Stressors in ICU: patients' evaluation. Intensive Care Med. 1997;23(12):1282-5.

5. Novaes MA, Knobel E, Bork AM, Pavão OF, Nogueira-Martins LA, Ferraz MB. Stressor in ICU: perception of the patient, relatives and health care team. Intensive Care Med. 1999;25:1421-6

6. Bitencourt AGV, Neves FBCS, Dantas MP, Albuquerque LC, De Melo RMV, Almeida AM, et al. Análise de estressores para o paciente em Unidade de Terapia Intensiva. Rev Bras Ter Intensiva. 2007;19(1):53-9.

7. Azoulay E, Pochard F, Chevret S, Lemaire F, Mokhtari M, Le Gall JR, et al. Meeting the needs of intensive care unit patient families: a multicenter study. Am J Respir Crit Care Med. 2001;163:135-9.

8. Lipp MEN. 0 stress no adulto e a necessidade de um diagnóstico preciso. In: Lipp MEN. Manual do inventário de sintomas de stress para adultos. 2. ed. São Paulo: Ed. Casa do Psicólogo; 2000

9. Hewitt J. Psycho-affective disorder in intensive care units: a review. J Clin Nurs. 2002;11:575-84

10. SUPPORT principal investigators. A controlled trial to improve care for seriously ill hospitalized patients. JAMA. 1995;274:1591-8.

11. Jones C, Griffiths RD. Social support and anxiety levels in relatives of critically ill patients. Br J Intensive Care. 1995;44-7.

12. Paparrigopoulos T, Melissaki A, Efthymiou A, Tsekou H, Vadala C, Kribeni G, et al. Shortterm psychological impact on family members of intensive care unit patients. J Psychos Res. 2006;61:719-22.

13. Gries CJ, Engelberg RA, Kross EK, Zatzick D, Nielsen EL, Downey L, et al. Predictors of symptoms of posttraumatic stress and depression in family members after patient death in the ICU. Chest. 2010;137(2):280-7.

14. Jones C, Skirrow P, Griffiths RD, Humphris G, Ingleby S, Eddleston J, et al. Post-traumatic stress disorder-related symptoms in relatives of patients following intensive care. Intensive Care Med. 2004;30:456-60.
15. Azoulay E, Pochard F, Kentish-Barnes N, Chevret S, Aboab J, Adrie C, et al. Risk of posttraumatic stress symptoms in family members of intensive care unit patients. Am J Respir Crit Care Med. 2005;171:987-94.

16. Auerbach SM, Kiesler DJ, Wartella J, Rausch S, Ward KR, Ivatury R. Optimism, satisfaction with needs met, interpersonal perceptions of the healthcare team, and emotional distress in patients' family members during critical care hospitalization. Am J Crit Care. 2005;14:202-10.

17. Davidson JE, Powers K, Hedayat KM, Tieszen M, Kon AA, Shepard E, et al. Clinical practice guidelines for support of the family in the patient-centered intensive care unit: American College of Critical Care Medicine Task Force 2004-2005. Crit Care Med. 2007;35(2):605-22.

18. Ballard KS. Identification of environmental stressors for patients in a surgical intensive care unit. Issues Ment Health Nurs. 1981;3:89-108.

19. Nastasy EL. Identifying environmental stressors for cardiac surgery patients in SICU. In: Proceedings of the $12^{\text {th }}$ Annual National Teaching Institute of AACN (1985). Newport Beach, Calif: AACN 357.

20. Azoulay E, Pochard F, Kentish-Barnes N, Chevret S, Aboab J, Adrie C, et al. Risk of posttraumatic stress symptoms in family members of intensive care unit patients. Am J Respir Crit Care Med. 2005;171:987-94.

21. Azoulay E, Pochard F, Chevret S, et al. Family participation in care to the critically ill: opinions of families and staff. Intensive Care Med. 2003;29:1498-504.

22. Dodek PM, Heyland DK, Graeme RM, Cook DJ. Translating family satisfaction data into quality improvement. Crit Care Med. 2004;32:1922-7.

23. Marosti CA, Dantas RAS. Relação entre estressores e características sociodemográficas e clínicas de pacientes internados em uma unidade coronariana. Rev Lat Am Enferm. 2006;14(5):713-9.

24. Correa AK, Sales, CA, Soares L. A família do paciente internado em terapia intensiva: concepções do enfermeiro. Acta Sci. 2002;24:811-8.

25. Souza RMC. Visitas em UTI: subsídios para reflexão. Rev Paul Hosp. 1988;36:24-9.

26. Plowright Cl. Needs of visitors in the intensive care unit. Br J Nurs. 1995;4(18):1081-3.

27. Byock I. Caring well of the families of our patients. Chest. 2007;132(5):1420-1.

28. Fumis RRL. As famílias dos pacientes da UTI do Hospital do Câncer - A. C. Camargo: suas necessidades e compreensão [dissertação]. São Paulo (SP): Fundação Antônio Prudente; 2004.

29. Neves FBCS, Dantas MP, Bitencourt AGV, Vieira PS, Magalhães LT, Teles JMM, et al. Análise da satisfação dos familiares em unidade de terapia intensiva. Rev Bras Ter Intensiva. 2009:21(1):32-7.

30. Vandall-Walker V, Jensen L, Oberle K. Nursing support for family members of critically ill adults. Qual Health Res. 2007:17(9):1207-18

31. Jamerson PA, Scheibmeir M, Bott MJ, Crighton F, Hinton RH, Cobb AK. The experiences of families with a relative in the intensive care unit. Heart Lung. 1996;25(6):467-74.

32. Heyland DK, Tranmer JE. Measuring family satisfaction with care in the Intensive Care Unit: The development of a questionaire and preliminary results. J Crit Care. 2001;16(4):142-9.

33. Mularski RA, Heine CE, Osborne ML, Ganzini L, Curtis JR. Quality of dying in the ICU: ratings by family members. Chest. 2005;128:280-7. 